\title{
Mining the deep Red-Sea brine pool microbial community for anticancer therapeutics
}

\author{
Luke Esau', Guishan Zhang ${ }^{2,3}$, Sunil Sagar ${ }^{1}$, Ulrich Stingl ${ }^{3,4}$, Vladimir B. Bajic ${ }^{1}$ and Mandeep Kaur ${ }^{1,5^{*}}$ (D)
}

\begin{abstract}
Background: Microbial species in the brine pools of the Red Sea and the brine pool-seawater interfaces are exposed to high temperature, high salinity, low oxygen levels and high concentrations of heavy metals. As adaptations to these harsh conditions require a large suite of secondary metabolites, these microbes have a huge potential as a source of novel anticancer molecules.

Methods: A total of 60 ethyl-acetate extracts of newly isolated strains from extreme environments of the Red-Sea were isolated and tested against several human cancer cell lines for potential cytotoxic and apoptotic activities.

Results: Isolates from the Erba brine-pool accounted for 50\% of active bacterial extracts capable of inducing 30\% or greater inhibition of cell growth. Among the 60 extracts screened, seven showed selectivity towards triple negative BT20 cells compared to normal fibroblasts.

Conclusion: In this study, we identified several extracts able to induce caspase-dependent apoptosis in various cancer cell lines. Further investigations and isolation of the active compounds of these Red Sea brine pool microbes may offer a chemotherapeutic potential for cancers with limited treatment options.
\end{abstract}

Keywords: Deep Red-Sea, Triple negative breast cancer, Brine pools, Anticancer

\section{Background}

The hallmarks of cancer, which categorises the survival and proliferative mechanisms of cancer cells, was first described by Hanahan and Weinberg [1]. Although these aspects of cancer are well studied, and even with the advances in personalized medicine for patient tailored treatment, there still exists cancer forms for which limited treatment options are available. Breast cancer, the most prevalent cancer in women globally, has an estimated 1.67 million cases diagnosed in 2012 [2]. Women of Arab [3] and African descent [4] present breast cancer at an earlier age, have more aggressive tumour types with a high prevalence of triple negative breast cancer. Penta-negative tumours (i.e., negative for

\footnotetext{
* Correspondence: mandeep.kaur@wits.ac.za

${ }^{1}$ King Abdullah University of Science and Technology (KAUST),

Computational Bioscience Research Center (CBRC), Thuwal, Jeddah

23955-6900, Saudi Arabia

${ }^{5}$ School of Molecular and Cell Biology, University of the Witwatersrand,

Johannesburg 2050, South Africa

Full list of author information is available at the end of the article
}

the estrogen and progesterone receptors, EGFR, HER2, and cytokeratin 5/6) have also been reported in Saudi women [5]. In our previous seminal investigations [6, 7], we reported the anticancer potential of extracts obtained from brine-pool microbes. Most of these extracts were active against estrogen receptor positive $(E R+)$ breast cancer cells. Since the triple-negative type of breast cancer is a more aggressive form and as currently no therapies are available, we aimed to identify new sources of anticancer compounds that can pave the way to develop novel therapies for triple negative breast cancer.

Approximately $60 \%$ of current anticancer therapeutics are derived from natural products, including, for example, marine-derived compounds such as cytarabine, trabectedin, eribulin, and dolastatins [8]. A review by Agrawal et al. [9] described nonribosomal peptides isolated from marine microbes having anticancer activity while in a research article Neelam et al. [10] discovered a marine halo-alkaliphilic bacteria species possessing anti breast cancer activity. This evidence supports the

(c) The Author(s). 2019 Open Access This article is distributed under the terms of the Creative Commons Attribution 4.0 International License (http://creativecommons.org/licenses/by/4.0/), which permits unrestricted use, distribution, and reproduction in any medium, provided you give appropriate credit to the original author(s) and the source, provide a link to the Creative Commons license, and indicate if changes were made. The Creative Commons Public Domain Dedication waiver (http://creativecommons.org/publicdomain/zero/1.0/) applies to the data made available in this article, unless otherwise stated. 
pursuit of mining marine environments for the discovery of new anticancer agents. Twenty-five (mostly) anaerobic deep-sea brine pools with extremely high salt concentrations have been reported in the Red Sea. Multiextremophilic microbes that inhabit these environments are not only adapted to high salinity (4-26\%), but also to elevated temperature, low oxygen concentrations, and high concentrations of heavy metals [11-14]. These extreme marine environmental conditions favour the production of secondary metabolites and thus potentially unique and potent natural compounds. Extremophilic marine bacterial species from these environments, therefore, present a unique opportunity for discovering novel anticancer compounds [6, 7] that address the everchanging need for improved chemotherapeutic drugs.

This study reports on anticancer activity of extracts from bacterial isolates from different habitats around brine pools within the Red Sea. We screened a total of 60 extracts against seven cell lines representing colorectal carcinoma, fibrosarcoma, breast carcinoma, cervical carcinoma, neuroblastoma, and normal cell lines. After initial screening, active extracts ( $>30 \%$ growth inhibition) were selected for testing in selected cell lines to investigate if apoptotic activities were moderated by caspases (executors of apoptotic cell death).

\section{Methods}

\section{Field sampling}

The inoculum for microbial isolations were collected during a cruise between Oct 16 and Nov 32011 as described in Sagar et al. [7].

\section{Source of bacterial isolates}

Sixty bacterial strains were isolated from different habitats in or around the brine pools (Table 1). While the deep-sea brine pool habitats differ in their physicochemical characteristics [15], several studies showed that all of these environments harbour high microbial diversity and biomass.

\section{PCR amplification}

DNA extraction, PCR amplification, and bioinformatics analyses of 16S rRNA genes from biomass of bacterial strains was performed according to Sagar et al. [7]. Sequences were deposited in Genbank, and accession numbers are listed in Table 1.

\section{Bacterial biomass}

The inocula were streaked using three different media types as described by (Sagar et al. 2013 [6, 7]). These solidified media types were supplemented with either $10 \%$ or $15 \%$ or $20 \%$ or $26 \% \mathrm{NaCl}(\mathrm{w} / \mathrm{v})$ before autoclaving to mimic salt concentrations in their original habitat. All incubations were done in a Binder incubator (Type
BD53, Binder, Tuttlingen, Germany). Strains reported here were isolated under oxic conditions (air, 21\% $\mathrm{O}_{2}$ ) and atmospheric pressure (1 atm) described elsewhere $[16,17]$. To collect biomass, all strains were grown for 2 to 3 weeks with constant agitation at $30^{\circ} \mathrm{C}$ in $5.0 \mathrm{l}$ of Marine Broth (Difco) supplemented with the respective concentration of $\mathrm{NaCl}$. Bacterial biomass was harvested and ethyl acetate extracts were prepared according to Sagar et al. [6, 7].

\section{Cell culturing}

BJ (Fibroblast), HCT (Colorectal adenocarcinoma), HT1080 (Fibrosarcoma), MCF-7 (Breast Adenocarcinoma), IMR-32 (Neuroblastoma), BT20 (Breast Adenocarcinoma), and HeLa (Cervical carcinoma) were obtained from the American Type Cell Culture Collection (ATCC, Manassas, VA). The cells were cultured in DMEM (Dulbecco's Modified Eagle's Medium) containing 10\% FCS (Foetal calf serum), and streptomycin $(100 \mu \mathrm{g} / \mathrm{mL})$ and penicillin $(100 \mathrm{U} / \mathrm{mL})$ in a $37^{\circ} \mathrm{C}$ incubator supplying $5 \% \mathrm{CO}_{2}$.

\section{MTT assay}

$2.5 \times 10^{3}$ cells were seeded per well in 384-well culture plates and treated with $200 \mu \mathrm{g} / \mathrm{mL}$ marine bacterial extracts for $48 \mathrm{~h}$. Growth inhibitory effects of extracts were estimated by an MTT (3-(4, 5-Dimethylthiazol-2yl)-2,5-diphenyltetrazolium bromide) assay as previously described [7]. A microtiter plate reader (BMG LabtechPHERAstar FS, Germany) was used to measure the OD (optical density) at $595 \mathrm{~nm}$ and the results were analyzed using Microsoft Office Excelఠ.

\section{APOPercentage assay}

Cells were seeded in quadruplicates in 96 well plates at a density of $5 \times 10^{3}$ cells per well in $90 \mu \mathrm{L}$ of media. After $24 \mathrm{~h}, 200 \mu \mathrm{g} / \mathrm{mL}$ extracts were added to the cells for $48 \mathrm{~h}$ with, while $30 \mathrm{~min}$ treatment with $10 \mathrm{mM} \mathrm{H}_{2} \mathrm{O}_{2}$ was used as a positive control. The cells were lifted and stained with the APOPercentage dye (Biocolor, UK), and analysed as described previously [18].

\section{Caspase-3/7 activity assay}

$2.5 \times 10^{3}$ cells were seeded in $20 \mu \mathrm{L}$ of media in 384 -well plates and allowed to settle overnight. Five microliters of extract $(200 \mu \mathrm{g} / \mathrm{mL})$ was added and further incubated for $48 \mathrm{~h}$. Manufacturer's instructions were followed to estimate Caspase-3/7 activity by using ApoTox-Glo kit (Promega) and the luminescence was measured using BMG LabtechPHERAstar FS (Germany). The results were normalized to cell viability (measured using MTT assay). 
Table 1 Identification of microbial stains. Taxonomic identification based on $16 \mathrm{~S}$ rRNA gene analysis and the source of inoculum for 60 microbial strains

\begin{tabular}{|c|c|c|c|c|c|c|}
\hline Name & Source & $\begin{array}{l}\text { Salinity } \\
(w / v)\end{array}$ & Closest relative & $\begin{array}{l}\text { Similarity } \\
\text { (16S rRNA genes) }\end{array}$ & $\begin{array}{l}\text { Accession no. of } \\
\text { the strains }\end{array}$ & $\begin{array}{l}\text { Accessionno. of the } \\
\text { closest relatives }\end{array}$ \\
\hline SB9 & Discovery interface & $25 \%$ & Haloprofundus marisrubri & $100 \%$ & KJ999759 & FN594944 \\
\hline SB3 & Discovery interface & $25 \%$ & Haloferax prahovense & $97 \%$ & KJ999758 & NR_028165 \\
\hline SB29 & Discovery interface & $25 \%$ & Haloferax larsenii & $98 \%$ & KJ999757 & NR_028209 \\
\hline SA10 & Kebrit brine & $26 \%$ & Haloferax prahovense & $99 \%$ & MG563761 & NR_028165 \\
\hline ZGT108 & Erba interface & $10 \%$ & Ruegeria profundi & $100 \%$ & KP726355 & NR_029197 \\
\hline ZGT114 & Erba interface & $10 \%$ & Microbulbifer salipaludis & $98 \%$ & KP726357 & NR_025232 \\
\hline ZGT118 & Erba interface & $10 \%$ & Ruegeria marisrubri & $100 \%$ & KP726356 & NR_029197 \\
\hline SJ5A-1 & Erba interface & $10 \%$ & Ponticoccus marisrubri & $97 \%$ & KP726358 & NR_044174 \\
\hline SJ5B & Erba interface & $10 \%$ & Ponticoccus litoralis & $99 \%$ & MG764545 & NR_044174 \\
\hline$X \mid 10$ & Erba interface & $10 \%$ & Pseudoalteromonas mariniglutinosa & $99 \%$ & MG768922 & NR_028992 \\
\hline H106 & Erba interface & $10 \%$ & Idiomarina zobellii & $99 \%$ & MG768917 & NR_024892 \\
\hline 1 & Kebrit brine & $26 \%$ & Halomonas axialensis & $99 \%$ & MG768918 & NR_027219 \\
\hline 2 & Kebrit interface & $20 \%$ & Halomonas salina & $99 \%$ & MG768925 & NR_042050 \\
\hline 3 & Kebrit interface & $20 \%$ & Marinimicrobium haloxylanilyticum & $99 \%$ & MG768919 & GQ920839 \\
\hline 4 & Kebrit interface & $20 \%$ & Halobacillus kuroshimensis & $99 \%$ & MG768920 & NR_041262 \\
\hline 5 & Erba interface & $10 \%$ & Chromohalobacter israelensis & $99 \%$ & MG768921 & NR_025431 \\
\hline 6 & Erba sediment & $18 \%$ & Alteromonas halophila & $99 \%$ & MG768928 & EU583725 \\
\hline 7 & Erba sediment & $18 \%$ & Halomonas taeanensis & $98 \%$ & MG768923 & NR_043087 \\
\hline 8 & Erba sediment & $18 \%$ & Halobacillus locisalis & $99 \%$ & MG768924 & NR_025715 \\
\hline 9 & Erba interface & $10 \%$ & Alteromonas macleodii & $99 \%$ & MG768926 & Y18228 \\
\hline 10 & Erba interface & $10 \%$ & Salinivibrio costicola. & $99 \%$ & MG768927 & NR_028703 \\
\hline 11 & Erba interface & $10 \%$ & Halomonas denitrificans & $99 \%$ & MG768930 & NR_042491 \\
\hline 12 & Erba interface & $10 \%$ & Pontibacillus marinus & $99 \%$ & MG768932 & NR_043011 \\
\hline 13 & Nereus interface & $10 \%$ & Pseudoalteromonas mariniglutinosa & $98 \%$ & MG768936 & NR_028992 \\
\hline 14 & Nereus interface & $10 \%$ & Pseudoalteromonas flavipulchra & $99 \%$ & MG768933 & NR_025126 \\
\hline 15 & Nereus interface & $10 \%$ & Salinivibrio sharmensis & $99 \%$ & MG768935 & AM279734 \\
\hline 16 & Nereus interface & $10 \%$ & Halomonas hamiltonii & $100 \%$ & MG768937 & AM941396 \\
\hline 17 & Nereus interface & $10 \%$ & Salinicola salarius & $99 \%$ & MG768934 & NR_042490 \\
\hline 18 & Discovery interface & $15 \%$ & Alteromonas macleodii & $98 \%$ & MG768957 & Y18228 \\
\hline 19 & Discovery interface & $15 \%$ & Halomonas halophila & $99 \%$ & MG768941 & NR_042697 \\
\hline 20 & Discovery interface & $15 \%$ & Alteromonas macleodii & $97 \%$ & MG768929 & AM885870 \\
\hline 21 & Discovery interface & $15 \%$ & Pontibacillus chungwhensis & $98 \%$ & MG768940 & NR_025812 \\
\hline 22 & Nereus interface & $10 \%$ & Salinicola salarius & $98 \%$ & MG768944 & NR_042490 \\
\hline 23 & Nereus interface & $10 \%$ & Zunongwangia profunda & $99 \%$ & MG768951 & NR_043986 \\
\hline 24 & Nereus interface & $10 \%$ & Marinobacter flavimaris & $99 \%$ & MG768954 & NR_025799 \\
\hline 25 & Nereus interface & $10 \%$ & Chromohalobacter marismortui & $99 \%$ & MG768956 & X87222 \\
\hline 26 & Kebrit interface & $20 \%$ & Salinivibrio proteolyticus & $99 \%$ & MG768958 & NR_043536 \\
\hline 27 & Nereus interface & $10 \%$ & Halomonas meridiana & $99 \%$ & MG768959 & AF212217 \\
\hline 28 & Erba sediment & $18 \%$ & Chromohalobacter israelensis & $98 \%$ & MG768971 & NR_025431 \\
\hline 29 & Erba sediment & $18 \%$ & Salinivibrio siamensis & $99 \%$ & MG770368 & NR_041552 \\
\hline 30 & Erba sediment & $18 \%$ & Idiomarina seosinensis & $99 \%$ & MG770369 & NR_025826 \\
\hline 31 & Erba sediment & $18 \%$ & Pseudoalteromonas carrageenovora & $99 \%$ & MG770359 & NR_026220 \\
\hline 32 & Erba sediment & $18 \%$ & Pseudoalteromonas ruthenica & $99 \%$ & MG770372 & NR_025140 \\
\hline
\end{tabular}


Table 1 Identification of microbial stains. Taxonomic identification based on 165 rRNA gene analysis and the source of inoculum for 60 microbial strains (Continued)

\begin{tabular}{|c|c|c|c|c|c|c|}
\hline Name & Source & $\begin{array}{l}\text { Salinity } \\
(w / v)\end{array}$ & Closest relative & $\begin{array}{l}\text { Similarity } \\
\text { (16S rRNA genes) }\end{array}$ & $\begin{array}{l}\text { Accession no. of } \\
\text { the strains }\end{array}$ & $\begin{array}{l}\text { Accessionno. of the } \\
\text { closest relatives }\end{array}$ \\
\hline 33 & Erba sediment & $18 \%$ & Idiomarina baltica & $99 \%$ & MG770373 & NR_027560 \\
\hline 34 & Erba interface & $10 \%$ & Pontibacillus halophilus & $99 \%$ & MG770364 & NR_044532 \\
\hline 35 & Erba interface & $10 \%$ & Alteromonas macleodii & $99 \%$ & MG770376 & Y18228 \\
\hline 37 & Nereus interface & $10 \%$ & Donghicolae burneus & $99 \%$ & MG770367 & NR_043928 \\
\hline 38 & Nereus interface & $10 \%$ & Halomonas aquamarina & $99 \%$ & MG770380 & NR_042063 \\
\hline 39 & Discovery interface & $15 \%$ & Idiomarina loihiensis & $100 \%$ & MG770382 & NR_025119 \\
\hline 40 & Discovery interface & $15 \%$ & Idiomarina zobellii & $98 \%$ & MG770370 & NR_024892 \\
\hline 41 & Nereus interface & $10 \%$ & Halomonas shengliensis & $99 \%$ & MG770371 & NR_044099 \\
\hline 42 & Nereus interface & $10 \%$ & Vibrio communis & $99 \%$ & MG770378 & GU078673 \\
\hline 43 & Nereus interface & $10 \%$ & Bacillus halodurans & $99 \%$ & MG770383 & NR_025446 \\
\hline 44 & Nereus interface & $10 \%$ & Vibrio natriegens & $99 \%$ & MG770400 & NR_026124 \\
\hline 45 & Nereus interface & $10 \%$ & Alteromonas macleodii & $98 \%$ & MG770410 & AM885870 \\
\hline 46 & Nereus interface & $10 \%$ & Thalassospira tepidiphila & $100 \%$ & MG770411 & NR_041492 \\
\hline 47 & Kebrit brine & $26 \%$ & Halostagnicola alkaliphila & $99 \%$ & MG775659 & AB533256 \\
\hline 53 & Nereus interface & $10 \%$ & Salegentibacter mishustinae & $99 \%$ & MG770459 & NR_025820 \\
\hline 54 & Nereus interface & $10 \%$ & Marinilactibacillus psychrotolerans & $99 \%$ & MG770461 & NR_024794 \\
\hline 55 & Erba interface & $10 \%$ & Alcanivorax dieselolei & $99 \%$ & MG773786 & NR_043106 \\
\hline
\end{tabular}

\section{Statistical analysis}

The samples (untreated vs. treated) were compared by using Student's $t$-test and statistical significance was noted at $p<0.05$. A Z-score of $\geq 0.6$ indicated robustness of assays [15].

\section{Results}

Taxonomic classification of microbes isolated from the Red Sea

Most bacteria isolated from the four brine pools Erba, Discovery, Kebrit, and Nereus were closely related to known and well described halophilic species within Proteobacteria and Archaea (Table 1).

\section{Anticancer activities of isolates in a panel of cell lines}

A total of 60 extracts isolated from bacterial cultures from the four brine pools Erba, Discovery, Kebrait, and Nereus were screened for anticancer activity by determining cell growth inhibition through MTT assay (Table 2). Extracts induced varying levels of growth inhibition and were classified into five groups - empty circle $(<30 \%)$, quarter-moon ( $>30$ and $<40 \%)$, half-moon ( $>40$ and $<60 \%$ ), three-quarter-moon (> 60 and $<80 \%$ ) and full-moon (>80\%). The skin fibroblast cell line BJ was used as a normal cell line control for screening anticancer activity of the extracts. BJ cell growth was inhibited by approximately $50 \%$ of extracts while the growth of neuroblastoma cell line IMR-32 remained largely insensitive to the treatment. Interestingly, the growth of the triple negative breast cancer cell line BT20 was sensitive to the majority of the extracts.

Further analysis revealed that the majority of bacterial isolates inducing active growth inhibition (above 30\%) in this study set were isolated from the Erba brine pool (Fig. 1). Figure 1 represents the distribution of active bacterial extracts inducing greater than $30 \%$ growth inhibition found across the brine-pools.

\section{Apoptosis as mode of anticancer activity}

We assessed phosphatidylserine exposure in cancer cells treated with selected microbial extracts using APOPercentage assay and monitored change in caspase-3/7 activity to determine if extracts induced apoptosis. For this purpose, the extracts inducing $>30 \%$ growth inhibition were selected for apoptosis screening (Table 3). Due to the availability of limited amount of microbial extracts, we performed apoptosis and caspase-3/7 activity assays only on extracts active in breast cancer, cervical cancer, and fibrosarcoma cell lines. Again, BJ cells were used as a control to identify extracts with selective anticancer activity. Extracts induced apoptosis in all cell lines. However four (ZGT118, XI10, 7 and 13), six (ZGT118, XI10, 13, 16, 30 and 55), one (9), and three $(10,15$ and 22$)$ extracts selectively induced apoptosis in MCF-7, BT20, HeLa and H T-1080 cells compared to BJ cells, respectively. The extract number 7 was only active against MCF-7 cells, whereas extracts 16 and 55 were selectively active against BT20 cells when the apoptosis- 
Table 2 The percentage growth inhibition of various cell lines after treatment with extracts. Growth inhibition of one normal (BJ) and six cancer cell lines treated with $200 \mu \mathrm{g} / \mathrm{ml}$ microbial ethyl-acetate extract for $48 \mathrm{~h}$

\begin{tabular}{|c|c|c|c|c|c|c|c|c|c|c|}
\hline Fxtreat & & & & Cell lit & & & HCT & & & \\
\hline $\begin{array}{c}\text { Extrcat } \\
\text { SB99 }\end{array}$ & $\begin{array}{l}\text { BJ } \\
Q\end{array}$ & $\frac{\text { MCF-7 }}{0}$ & $\begin{array}{c}\text { BT20 } \\
\end{array}$ & HeLa & $\begin{array}{c}\text { HT-1080 } \\
\end{array}$ & $\begin{array}{c}\text { IMR-32 } \\
0\end{array}$ & HCT & & & \\
\hline SB3 & 0 & 0 & 0 & 8 & 0 & 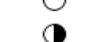 & 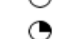 & 0 & $<30 \%$ & \\
\hline SB29 & 0 & 0 & 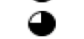 & 5 & 0 & 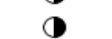 & 0 & 0 & $>30 \%$ & $<40^{\circ} \%$ \\
\hline SA10 & 5 & $\sigma$ & 0 & 0 & 0 & 0 & 0 & & $350 \%$ & $<40 \%$ \\
\hline ZGT108 & () & 0 & 0 & (1) & (1) & ( & C) & 0 & $>100$ & $<60^{\circ}$ \\
\hline ZGT114 & 임 & 0 & & ㅇ & 0 & 0 & 0 & 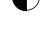 & $>40 \%$ & $<60 \%$ \\
\hline ZGT118 & 0 & 0 & - & (1) & 0 & C) & 0 & 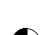 & & \\
\hline SJ5A-1 & ( & 0 & & (1) & (1) & 0 & () & 0 & $>60 \%$ & $<80 \%$ \\
\hline SJ5B & $\bullet$ & 0 & - & 0 & • & 0 & ( & & & \\
\hline XI10 & ( & ( & • & () & I & 1 & 1 & 0 & $>80 \%$ & \\
\hline H106 & - & - & 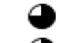 & (1) & - & 0 & C & & -8070 & \\
\hline 1 & 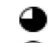 & 0 & 9 & 0 & 0 & 0 & C & & & \\
\hline 2 & 0 & (1) & 0 & (1) & (1 & 0 & $\odot$ & & & \\
\hline 3 & 0 & 0 & 0 & () & 0 & 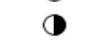 & (1) & & & \\
\hline 4 & 0 & 0 & 1 & 0 & 0 & 0 & 0 & & & \\
\hline 5 & ( & 0 & 0 & 0 & 0 & 0 & ( & & & \\
\hline 6 & 0 & C) & $\boldsymbol{\theta}$ & 0 & 0 & 0 & 0 & & & \\
\hline 7 & 0 & - & (1) & 0 & 0 & 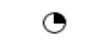 & 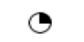 & & & \\
\hline 8 & 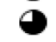 & C) & $\boldsymbol{0}$ & 0 & () & 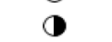 & 1 & & & \\
\hline 9 & 0 & 0 & (1) & 0 & 0 & C) & 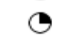 & & & \\
\hline 10 & 0 & 0 & 0 & 0 & () & 0 & 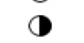 & & & \\
\hline 11 & 0 & 0 & 0 & 0 & 0 & 0 & ( & & & \\
\hline 12 & 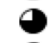 & 0 & 0 & 0 & (1) & 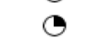 & (1) & & & \\
\hline 13 & () & C) & () & (1) & (1) & c) & 0 & & & \\
\hline 14 & 0 & 0 & C & () & ( & C) & 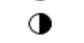 & & & \\
\hline 15 & ( & 0 & 0 & (1) & $\bullet$ & C) & 1 & & & \\
\hline 16 & C) & C) & & () & 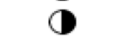 & 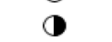 & 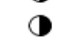 & & & \\
\hline 17 & 0 & 0 & (1) & $\bullet$ & () & 0 & 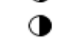 & & & \\
\hline 18 & 0 & C) & 0 & () & () & 0 & 1 & & & \\
\hline 19 & 0 & 0 & () & 0 & 0 & 1 & ( & & & \\
\hline 20 & 0 & 0 & $\boldsymbol{\theta}$ & 0 & ( & 0 & (1) & & & \\
\hline 21 & () & C) & () & () & () & 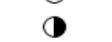 & 0 & & & \\
\hline 22 & () & (1) & 0 & () & (1) & 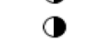 & 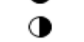 & & & \\
\hline 23 & 0 & 0 & C) & 0 & $C$ & 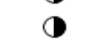 & 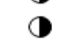 & & & \\
\hline 24 & 0 & () & 0 & C) & () & 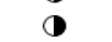 & 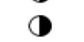 & & & \\
\hline 25 & () & () & & () & () & 0 & 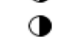 & & & \\
\hline 26 & 0 & 0 & & () & () & 1 & 0 & & & \\
\hline 27 & 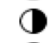 & 0 & & 6 & (1) & 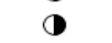 & (1) & & & \\
\hline 29 & 0 & 0 & $\boldsymbol{a}$ & 0 & 0 & $\bullet$ & (1) & & & \\
\hline 30 & ( & ( & 0 & ( & (1) & $\bullet$ & 0 & & & \\
\hline 31 & 0 & ( & 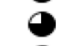 & 0 & 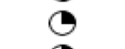 & () & - & & & \\
\hline 32 & 0 & 0 & ๑ & 0 & 0 & 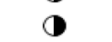 & $\bullet$ & & & \\
\hline 33 & 0 & ( & 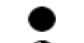 & () & () & (1) & 0 & & & \\
\hline 34 & 0 & 0 & 0 & 0 & 0 & 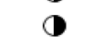 & 0 & & & \\
\hline 35 & 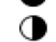 & 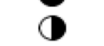 & 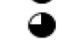 & 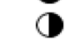 & (1) & 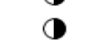 & 0 & & & \\
\hline 37 & 0 & $\boldsymbol{1}$ & & 0 & 6 & 1 & 0 & & & \\
\hline 38 & 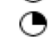 & 0 & & 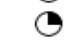 & $C$ & 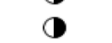 & $\bullet$ & & & \\
\hline 39 & (1) & (1) & 0 & () & () & 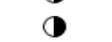 & 0 & & & \\
\hline 40 & () & 0 & 0 & () & () & 0 & 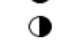 & & & \\
\hline 41 & () & 0 & () & () & () & 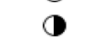 & $\bullet$ & & & \\
\hline 42 & 0 & () & () & () & () & 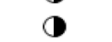 & $\bullet$ & & & \\
\hline 43 & 0 & 0 & 0 & 0 & 0 & 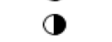 & 0 & & & \\
\hline 44 & $\bullet$ & • & () & 0 & () & 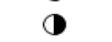 & 0 & & & \\
\hline 45 & 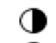 & (1) & (1) & 0 & (1) & (1) & 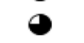 & & & \\
\hline 46 & 6 & 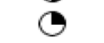 & 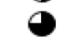 & () & () & 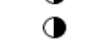 & 0 & & & \\
\hline 47 & C) & 0 & 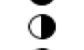 & 0 & 0 & $\bullet$ & 0 & & & \\
\hline 53 & 0 & 0 & () & () & () & 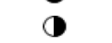 & $\bullet$ & & & \\
\hline 54 & 0 & 0 & $\boldsymbol{1}$ & 0 & 0 & 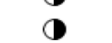 & 0 & & & \\
\hline 55 & () & 0 & & () & () & 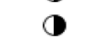 & 0 & & & \\
\hline
\end{tabular}




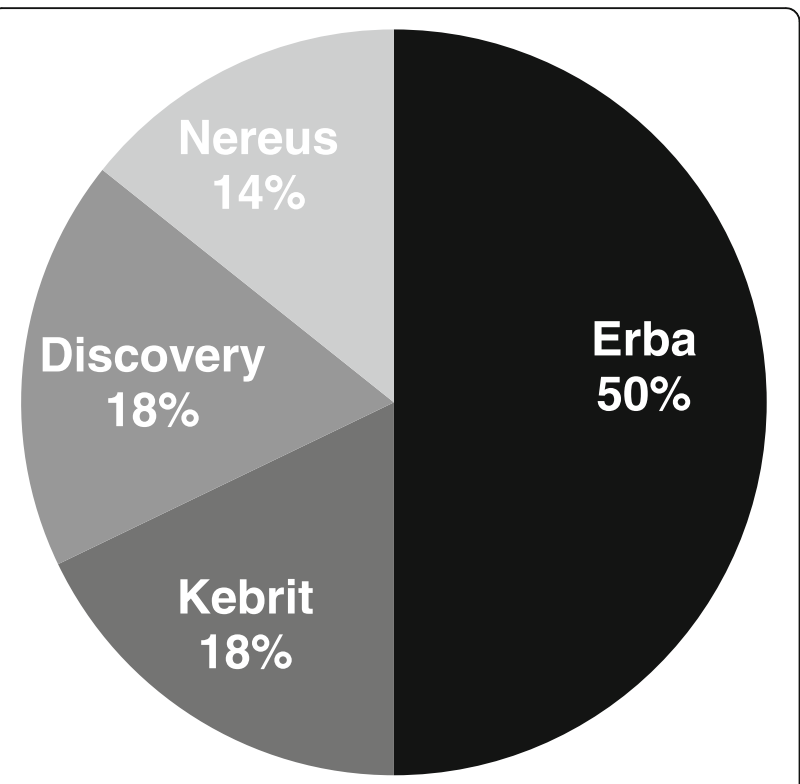

Fig. 1 The percentage of active extracts from bacterial strains isolated from the respective brine-pools. Pie chart represents the distribution of extracts based on their source in the Red Sea that induced greater than 30\% growth inhibition in various cancer cell lines at a concentration of $200 \mu \mathrm{g} / \mathrm{ml}$ after $24 \mathrm{~h}$ of treatment

inducing potential of these extracts was compared among all five cell lines (Table 3).

A Caspase-3/7 activity assay was employed to gain insights into the type of apoptosis occurring, namely caspase-dependent or caspase-independent (Fig. 2a-d). The caspase-3/7 results showed a clear trend that a caspase-independent mechanism was mostly responsible for BJ cell death. Extract number 7 induced caspasedependent apoptosis in MCF-7 cells but had no adverse effect on BJ cell death despite it inducing slight growth inhibition in BJ cells making it a promising drug candidate for future work. Extract number 19 induced caspase-dependent apoptosis in MCF-7, HeLa, and HT1080 cells but not in BJ cells, even though BJ cells stained positive for phosphatidylserine exposure by APOPercentage assay (Table 3). Caspase-3/7 activity increased in BT20 cells compared to BJ cells in response to extracts ZGT108, ZGT114, 12, 24, 37, 54 and 55. HeLa cells and HT1080 cells displayed significantly increased caspase-3/7 activation in response to extracts 4 and 19, respectively (Fig. 2). Interestingly, extract number 55 showed selectivity towards BT20 cells by inhibiting its growth and inducing apoptosis via caspase-3/7, neither of which was observed for BJ cells.

\section{Discussion}

As a follow-up from our previous studies [6, 7], we screened extracts from 60 marine bacteria isolated from brine pools of the Red Sea. We would like to emphasize that in our previous two investigations, we could only find extracts active against MCF-7 (ER+) cells, but the current study reports anticancer activities of the extracts isolated from the Red Sea brine-pool harboring bacteria against fibrosarcoma, cervical cancer and particularly BT20 (triple negative) cancer cells. Primary cytotoxicity screening of all extracts against seven cell lines representing five different cancers enabled us to broadly identify potential anticancer extracts from deep-sea microbes. All extracts were also screened against one normal fibroblast cell line (BJ) to further identify those who exhibited selectivity for cancer, but not normal cells.

We investigated whether the brine-pool location had any correlation with anticancer activity induced by the bacterial isolates. Of all isolates from the respective brine-pools, we found that about $50 \%$ of all isolates collected from Erba Deep (located at a depth of $2395 \mathrm{~m}$ ), showed greater than $30 \%$ growth inhibition (Fig. 1). The most closely related validly described species to these strains are (Table 1): Chromohalobacter israelensis, Salinivibrio siamensis, Idiomarina seosinensis, Pseudoalteromona scarrageenovora, Pontibacillus halophilus, and Alteromonas macleodii. Our previous work [7] has reported anticancer activity of Chromohalobacter israelensis in HeLa cells. A PubMed search did not reveal even a single article that describes the anticancer activity of any of the other five bacterial species. This highlights the fact that the microbes found in the deep-sea brine pools of the Red Sea (especially Erba Deep) may have unique anticancer compounds that can be explored in the future to develop new drugs.

Apoptosis assays (APOPercentage and caspase-3/7 activity) confirmed that several of these selected (showing $>30 \%$ cell growth inhibition) extracts induced apoptotic cell death in cancer cell lines. This secondary screening process identified the extracts that selectively targeted a specific type of cancer via apoptotic cell death. We further investigated if extracts from a particular bacterial species have anticancer activity towards a specific cell line. Intriguingly, all three extracts $(10,15$ and 22) that specifically inhibited the growth of fibrosarcoma cells (HT-1080) belonged to strains that were closely related to Salinivibrio costicola, Salinivibrio sharmensis, and Salinicola salaries, respectively, showing an enrichment of genus Salinivibrio. It would be interesting in future to investigate Salinivibrio extracts against other sarcomas as well. In MCF-7 cells, two out of four most active extracts (XI10 and 13) belonged to Pseudoalteromonas mariniglutinosa. This bacterial species had not been tested so far for anticancer activities (PubMed search). Secondary metabolites isolated from Psuedoalteromonas sp. off the coast of Brazil were reported to have potent anticancer activity against a leukemic and melanoma cell line, and the active compound, prodigiosin, was shown 
Table 3 The percentage apoptotic cell death induced by selected extracts in various cell lines. Heat map of extracts $(200 \mu \mathrm{g} / \mathrm{ml})$ inducing apoptosis in one normal (BJ) and selected cancer cell lines after $48 \mathrm{~h}$ treatment

\begin{tabular}{|c|c|c|c|c|c|}
\hline Extract & BJ & MCF-7 & BT20 & HeLa & HT1080 \\
\hline ZGT108 & 63.1 & -7.4 & 75.5 & 25.6 & 10.2 \\
\hline ZGT114 & 73.5 & 11.0 & 78.4 & 45.0 & 13.6 \\
\hline ZGT118 & 10.2 & 35.1 & 76.1 & -12.4 & 0.0 \\
\hline XI10 & -3.2 & 19.4 & 59.1 & -9.3 & 0.3 \\
\hline H106 & 56.1 & 27.0 & 42.4 & 18.7 & 14.0 \\
\hline 1 & 67.9 & -8.1 & -8.4 & -3.7 & 19.2 \\
\hline 2 & 53.7 & 6.1 & 25.1 & 62.4 & 1.9 \\
\hline 3 & 75.2 & -2.9 & 20.8 & 28.3 & 52.4 \\
\hline 4 & 72.4 & -7.1 & -7.8 & 53.9 & 22.4 \\
\hline 5 & 45.2 & -5.2 & 6.0 & -10.6 & 48.9 \\
\hline 7 & -7.1 & 37.5 & -6.3 & -24.4 & 0.5 \\
\hline 9 & 4.9 & -5.1 & -7.7 & 26.9 & 5.0 \\
\hline 10 & 8.1 & 11.1 & -0.8 & -5.3 & 54.7 \\
\hline 11 & 62.8 & -5.8 & 11.5 & 33.4 & 74.9 \\
\hline 12 & 59.6 & 11.5 & 65.5 & -9.5 & 22.6 \\
\hline 13 & 3.7 & 23.0 & 74.7 & -9.4 & 8.6 \\
\hline 14 & 30.3 & -9.2 & 20.9 & -10.9 & 3.7 \\
\hline 15 & 14.1 & -1.6 & -1.4 & 10.5 & 58.8 \\
\hline 16 & 4.3 & -2.3 & 25.8 & 0.2 & 2.6 \\
\hline 18 & 79.6 & -4.8 & -4.6 & -0.8 & 38.5 \\
\hline 19 & 72.7 & 42.8 & 8.0 & 45.2 & 20.8 \\
\hline 22 & 12.1 & -4.7 & 3.4 & 3.5 & 50.3 \\
\hline 23 & 76.9 & -0.5 & 2.4 & 9.8 & 45.5 \\
\hline 24 & 78.9 & 3.3 & 56.6 & 27.4 & 57.3 \\
\hline 30 & 4.7 & -1.8 & 20.9 & -13.1 & -0.3 \\
\hline 31 & 77.6 & 0.4 & 66.9 & 24.5 & 1.4 \\
\hline 34 & 73.2 & -4.7 & 4.0 & 6.7 & 29.6 \\
\hline 37 & 79.1 & -2.5 & 25.6 & 30.2 & 19.6 \\
\hline 40 & 18.6 & -7.1 & 80.4 & 4.7 & 2.8 \\
\hline 41 & 88.4 & 15.4 & 82.3 & 22.1 & 63.1 \\
\hline 42 & 88.2 & 0.1 & 81.6 & 7.7 & 4.5 \\
\hline 45 & 30.4 & -7.7 & 73.9 & -6.0 & -2.2 \\
\hline 47 & 85.5 & -3.0 & 75.0 & 4.3 & 4.2 \\
\hline 53 & 88.3 & -2.6 & 46.5 & 2.7 & 2.1 \\
\hline 54 & 88.1 & -4.9 & 80.3 & 6.6 & 3.2 \\
\hline 55 & 4.2 & -2.6 & 26.7 & -10.9 & -4.7 \\
\hline
\end{tabular}

\% Apoptosis

$0 \% \quad>20 \% \quad>40 \%$


A

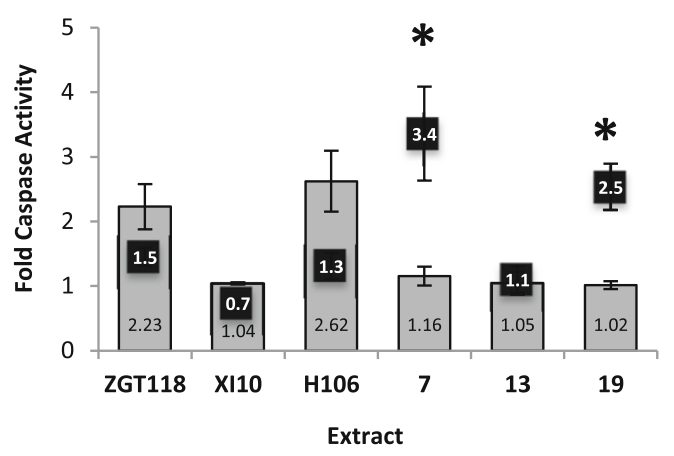

B

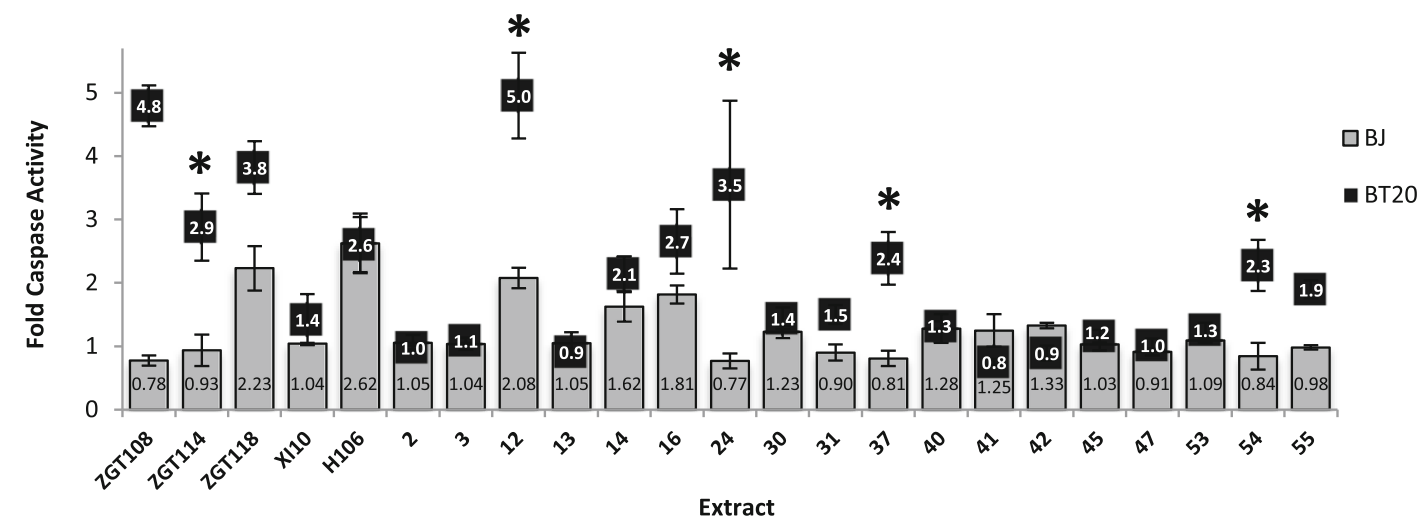

C

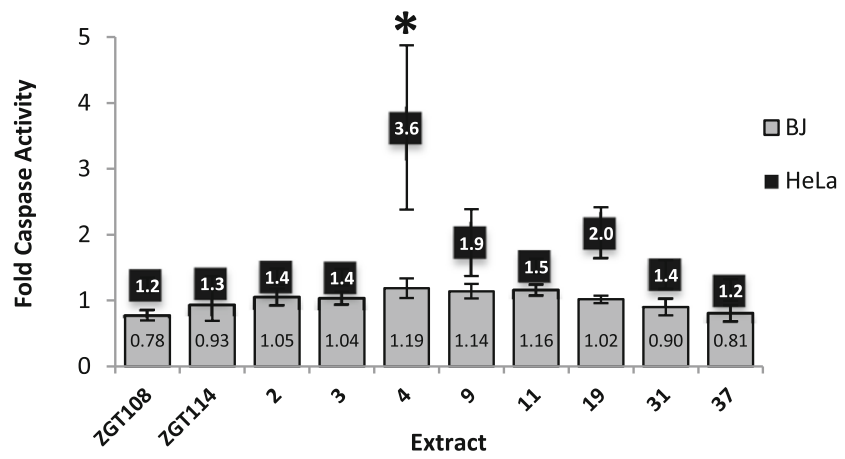

D

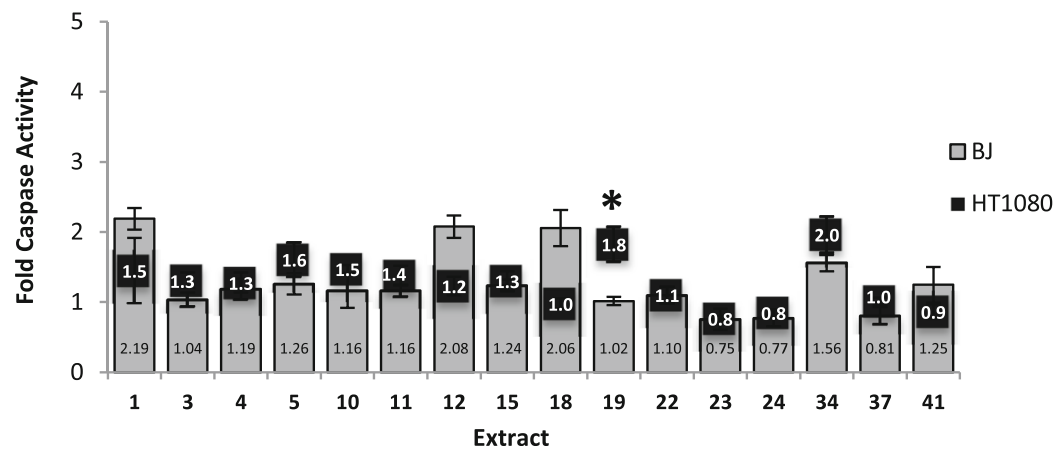

Fig. 2 Caspase 3/7 Activity in cells treated with bacterial extract. Normal skin fibroblast cell line BJ and four cancer cell lines MCF-7, BT20, HeLa and HT1080 were treated with $200 \mathrm{\mu g} / \mathrm{ml}$ bacterial extract for $48 \mathrm{~h}$ (a-d). Fold change in Caspase-3/7 activity relative to untreated was determined as per manufacturer's instructions and students t-test was used to determine significance where ${ }^{*}$ indicates $p<0.05$ 
to be selective for cancers overexpressing ErbB-2 [19]. In BT20 cells, extracts (16 and 55) showed selective cell death, and these extracts belong to species Halomonas hamiltonii and Alcanivorax dieselolei, respectively, none of which have ever been shown to have anticancer activity before. Our observation that Halomonas sp. and Alteromonas sp. contributed to the majority of growth inhibition may be attributed to their ability to produce molecules including exopolysaccharides (EPSs) and Dithiolopyrrolone (DTP), respectively. Exopolysaccharides (heterogeneous polymers) isolated from Halomonas stenophila and Halomonas smyrnensis induced proapoptotic effects against human T-leukemia cells [20] and breast cancer MCF-7 cells [21]. Alteromonas sp. are also well known for producing dithiolopyrrolone (DTP) molecules, which are known potent natural antibiotics; DTP obtained FDA approval as topical antibiotic Bactroban $^{\circ}$ (GlaxoSmithKline) [22]. Not surprising, DTP also exhibits potent anticancer activity. These classes of antibiotic or polysaccharide type molecules most probably explain the anticancer activity we observed in this study.

In conclusion, our study has identified several microbial species that have the potential to kill selectively cancer cells, and interestingly many of these species have never been previously tested for their anticancer activities. Here, we provide seminal baseline data pinpointing which bacterial species and brine-pools should be targeted for future investigations to isolate anticancer compounds. This work is of particular importance for triple negative breast cancer therapeutic development as no drug exists till date that can effectively cure this aggressive form of breast cancer. Testing of these marine extracts against penta-negative breast cancer cells should be of great interest to future studies.

\section{Abbreviations}

DMEM: Dulbecco's Modified Eagle's Medium; DTP: Dithiolopyrrolone; ER: Estrogen receptor; FCS: Foetal calf serum; MTT: 3-(4, 5-Dimethylthiazol-2yl)-2, 5-diphenyltetrazolium bromide; OD: Optical density;

PS: Phosphatidylserine

\section{Acknowledgements}

Authors want to thank Dr. Andre Antunes and Dr. Tyas Hikmawan for collecting samples.

\section{Authors' contributions}

LE and MK performed biological testing experiments and wrote the manuscript. LE analysed data and compiled tables/figures. SS prepared the extracts for biological evaluations and planned the study with MK. GZ grew the strains in large batches, isolated the strains and provided the taxonomic classification. US planned the exhibition and the cultivation experiments along with the writing of the manuscript. VBB provided general coordination of the study. All authors read and approved the final manuscript.

\section{Funding}

Authors thank SEDCO for funding this research.

\section{Availability of data and materials}

All 16S rRNA gene sequences of newly isolated strains from this study have been deposited in Genbank. Please refer to Table 1 for accession numbers.
Ethics approval and consent to participate

Not applicable.

\section{Consent for publication}

Not applicable.

\section{Competing interests}

The authors declare that they have no competing interests.

\section{Author details}

${ }^{1}$ King Abdullah University of Science and Technology (KAUST), Computational Bioscience Research Center (CBRC), Thuwal, Jeddah 23955-6900, Saudi Arabia. ${ }^{2}$ Key Laboratory of Microbial Resources Collection and Preservation, Ministry of Agriculture, Institute of Agricultural Resources and Regional Planning, Chinese Academy of Agricultural Sciences, Beijing 100081, People's Republic of China. ${ }^{3}$ King Abdullah University of Science and Technology, Red Sea Research Center, Thuwal, Jeddah 23955-6900, Saudi Arabia. ${ }^{4}$ Department of Microbiology and Cell Science, Institute of Food and Agricultural Sciences, University of Florida, UF/IFAS Fort Lauderdale Research and Education Center, Davie, FL 33314, USA. ${ }^{5}$ School of Molecular and Cell Biology, University of the Witwatersrand, Johannesburg 2050, South Africa.

Received: 24 November 2017 Accepted: 9 June 2019

Published online: 20 June 2019

\section{References}

1. Hanahan D, Weinberg RA. Hallmarks of cancer: the next generation. Cell. 2011;144(5):646-74.

2. Ferlay J, Soerjomataram I, Dikshit R, Eser S, Mathers C, Rebelo M, Parkin DM, Forman D, Bray F. Cancer incidence and mortality worldwide: sources, methods and major patterns in GLOBOCAN 2012. Int J Cancer. 2015;136(5):E359-86.

3. Chouchane L, Boussen H, Sastry KS. Breast cancer in Arab populations: molecular characteristics and disease management implications. Lancet Oncol. 2013;14(10):e417-24.

4. Stead LA, Lash TL, Sobieraj JE, Chi DD, Westrup JL, Charlot M, Blanchard RA, Lee JC, King TC, Rosenberg CL. Triple-negative breast cancers are increased in black women regardless of age or body mass index. Breast Cancer Res. 2009;11(2):R18.

5. Al Tamimi DM, Shawarby MA, Ahmed A, Hassan AK, AlOdaini AA. Protein expression profile and prevalence pattern of the molecular classes of breast cancer- a Saudi population based study. BMC Cancer. 2010;10:223.

6. Sagar S, Esau L, Hikmawan T, Antunes A, Holtermann K, Stingl U, Bajic VB, Kaur M. Cytotoxic and apoptotic evaluations of marine bacteria isolated from brine-seawater interface of the Red Sea. BMC Complement Altern Med. 2013;13:29

7. Sagar S, Esau L, Holtermann K, Hikmawan T, Zhang G, Stingl U, Bajic VB, Kaur M. Induction of apoptosis in cancer cell lines by the Red Sea brine pool bacterial extracts. BMC Complement Altern Med. 2013;13:344.

8. Dyshlovoy SA, Honecker F. Marine compounds and cancer: where do we stand? Mar Drugs. 2015:13(9):5657-65.

9. Agrawal S, Acharya D, Adholeya A, Barrow CJ, Deshmukh SK. Nonribosomal peptides from marine microbes and their antimicrobial and anticancer potential. Front Pharmacol. 2017:8:828.

10. Neelam DK, Agrawal A, Tomer AK, Bandyopadhayaya S, Sharma A, Jagannadham MV, Mandal CC, Dadheech PK. A Piscibacillus sp. isolated from a soda Lake exhibits anticancer activity against breast cancer MDAMB-231 cells. Microorganisms. 2019;7(2). https://doi.org/10.3390/ microorganisms7020034

11. Antunes A, Ngugi DK, Stingl U. Microbiology of the Red Sea (and other) deep-sea anoxic brine lakes. Environ Microbiol Rep. 2011;3(4):416-33.

12. Craig H. Isotopic composition and origin of the red sea and salton sea geothermal brines. Science. 1966;154(3756):1544-8.

13. Hartmann M, Scholten JC, Stoffers P, Wehner F. Hydrographic structure of brine-filled deeps in the Red Sea-new results from the Shaban, Kebrit, Atlantis II, and Discovery Deep. Mar Geol. 1998;144(4):311-30.

14. Schmidt M, Botz R, Faber E, Schmitt M, Poggenburg J, Garbe-Schönberg D, Stoffers $P$. High-resolution methane profiles across anoxic brine-seawater boundaries in the Atlantis-II, Discovery, and Kebrit Deeps (Red Sea). Chem Geol. 2003;200(3):359-75. 
15. Zhang JH, Chung TD, Oldenburg KR. A simple statistical parameter for use in evaluation and validation of high throughput screening assays. J Biomol Screen. 1999;4(2):67-73.

16. Zhang G, Haroon MF, Zhang R, Dong X, Wang D, Liu Y, Xun W, Stingl U. Ruegeria profundi sp. nov. and Ruegeria marisrubri sp. nov., isolated from the brine-seawater interface at Erba Deep in the Red Sea. Int I Syst Evol Microbiol. 2017;67(11):4624-31.

17. Zhang G, Gu J, Zhang R, Rashid M, Haroon MF, Xun W, Ruan Z, Dong X, Stingl U. Haloprofundus marisrubri gen. nov., sp. nov., an extremely halophilic archaeon isolated from a brine-seawater interface. Int I Syst Evol Microbiol. 2017;67(1):9-16.

18. Kaur M, Esau L. Two-step protocol for preparing adherent cells for highthroughput flow cytometry. Biotechniques. 2015;59(3):119-26.

19. Arthaud ID, Rodrigues FA, Jimenez PC, Montenegro RC, Angelim AL, Maciel VM, Silveira ER, Freitas HP, Sousa TS, Pessoa OD, et al. Studies on the secondary metabolites of a Pseudoalteromonas $\mathrm{sp}$. isolated from sediments collected at the northeastern coast of Brazil. Chem Biodivers. 2012;9(2):418-27.

20. Ruiz-Ruiz C, Srivastava GK, Carranza D, Mata JA, Llamas I, Santamaria M, Quesada E, Molina IJ. An exopolysaccharide produced by the novel halophilic bacterium Halomonas stenophila strain B100 selectively induces apoptosis in human T leukaemia cells. Appl Microbiol Biotechnol. 2011;89(2): 345-55.

21. Queiroz E, Fortes ZB, da Cunha MAA, Sarilmiser HK, Barbosa Dekker AM, Oner ET, Dekker RFH, Khaper N. Levan promotes antiproliferative and proapoptotic effects in MCF-7 breast cancer cells mediated by oxidative stress. Int J Biol Macromol. 2017;102:565-70

22. Li B, Wever WJ, Walsh CT, Bowers AA. Dithiolopyrrolones: biosynthesis, synthesis, and activity of a unique class of disulfide-containing antibiotics. Nat Prod Rep. 2014;31(7):905-23.

\section{Publisher's Note}

Springer Nature remains neutral with regard to jurisdictional claims in published maps and institutional affiliations.

Ready to submit your research? Choose BMC and benefit from:

- fast, convenient online submission

- thorough peer review by experienced researchers in your field

- rapid publication on acceptance

- support for research data, including large and complex data types

- gold Open Access which fosters wider collaboration and increased citations

- maximum visibility for your research: over $100 \mathrm{M}$ website views per year

At $\mathrm{BMC}$, research is always in progress.

Learn more biomedcentral.com/submissions 